\title{
Trends and developments in home enteral tube feeding in Lothian
}

\author{
J. Mackel and K. Henderson \\ Community Enteral Nutrition Team, Department Nutrition and Dietetics, Royal Edinburgh Hospital, EH10 5HF, UK
}

In December 2005, the Community Enteral Nutrition Team (CENT) was established in Lothian to provide a specialist, equitable, panLothian dietetic service to adults receiving enteral tube feeding at home.

Prior to 2005 the dietetic management of patients on home enteral tube feeding (HETF) was inadequate and the team was established to improve the care provided to these patients. The number of patients receiving HETF in Lothian has increased by $45.6 \%$ since 2005 and our total number of patients is higher than the average of 66 patients per year per centre reported by BANS in $2007^{(1)}$.

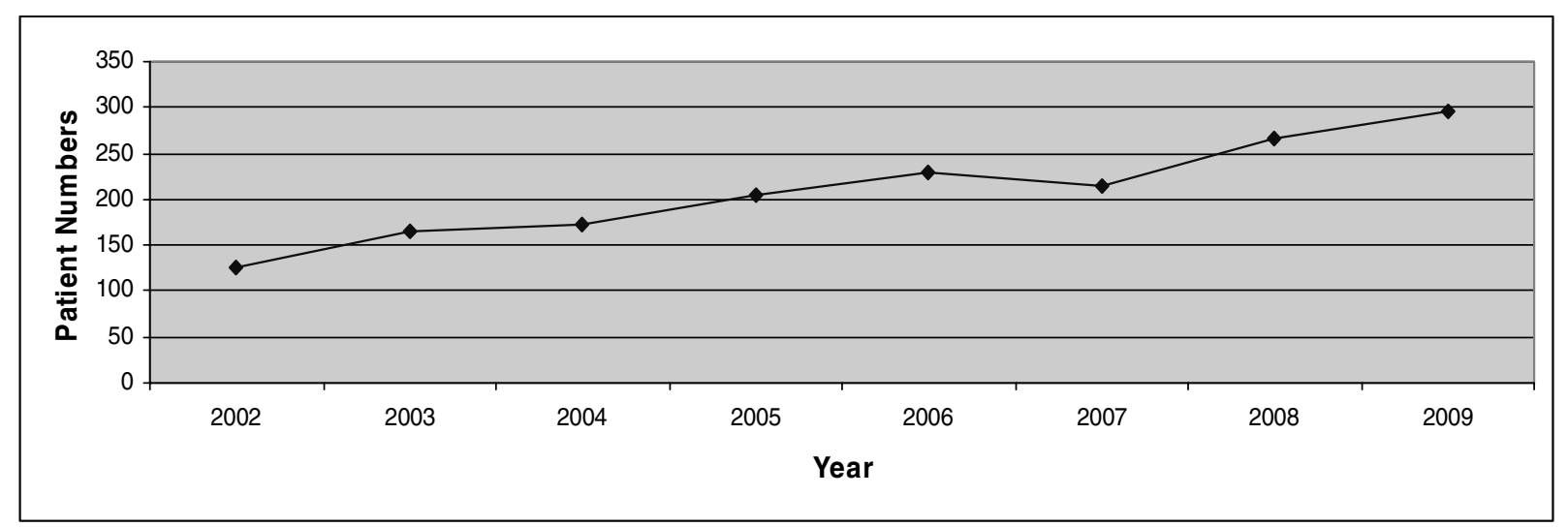

Within our current caseload $46.4 \%$ of patients have diseases of the central nervous system, $12.3 \%$ GI disease, $4.4 \%$ respiratory disease, $2.2 \%$ renal disease, $27.2 \%$ head and neck cancer and $7.5 \%$ others.

CENT works closely with dietetic colleagues at Royal Hospital for Sick Children to complete the transition of paediatric HETF patients into adult services. In 2009, a total of $19(6 \%)$ were in the transition process compared to 24 (9\%) in 2008. Five patients were transferred to adult services in 2008/09. CENT manages all 47 (15\%) enteral tube fed patients in care homes across Lothian.

The team currently works to a standard of visiting patients and their carers at home within 5 working days of their discharge from hospital and in $2009,70 \%$ of patients were seen within 5 working days.

In partnership with Homeward (Nutricia Clinical Care) in 2007 CENT carried out a patient satisfaction questionnaire (PSQ) and compared the results with a previous PSQ from 2005. The results showed:

- Improved patient satisfaction since the introduction of CENT.

- Patients would like more contact with a dietitian.

- Patients are satisfied with the home delivery service.

The PSQ is currently being conducted for 2009.

CENT co-ordinate and facilitate a number of recurring training initiatives for staff across Lothian to provide consistent, high quality training in line with Lothian Best Practice Statement ${ }^{(2)}$. Between July 2007 and June 2008 a total of 148 trained staff and 150 support workers attended the training courses.

With the numbers of patients on HETF continuing to increase CENT is constantly redesigning delivery of patient care and has evolved into a highly specialised dietetic resource providing a quality service to patients, carers and other health care professionals in Lothian.

1. BANS (2007) Artificial Nutrition Support in the UK 2000-2007 (published on the BAPEN website 2008, http://www.bapen.org.uk (ISBN 1899467165)

2. NHS Lothian (2007) Lothian Enteral Tube Feeding Best Practice Statement for Adults and Children 